\title{
What is special about the brains of musicians?
}

\author{
Lutz Jäncke
}

Department of Neuropsychology, University of Zürich, Switzerland

Musicians form an ideal and interesting subject pool to study structural and functional adaptations associated with exceptional cognitive and motor performance. To understand this, one has to imagine a typical career of a professional musician. One outstanding feature of musically talented children who progress to become outstanding performers is their conscientious approach to practicing their instrument at ages which could obviously influence brain development. This was highlighted by Ericson et al. [1], who found that accumulated practice time for the best violin students at the Berlin Academy was $7400 \mathrm{~h}$ by the time they were 18 years old, and for expert pianists it was $7600 \mathrm{~h}$. This is in contrast to $3400 \mathrm{~h}$ for violinists who were student music teachers and to $1600 \mathrm{~h}$ for amateur pianists. Ericson also reported that the highest achievers practiced at the same time of day and for a consistent amount of time. These findings are supported by those of Sloboda and Davidson [2], who reported a positive correlation between amount of practice and grade achievement in Associated Board of Royal Schools of Music practical examinations. Even with novice musicians, amount of practice can predict musical outcomes as reported in a study of 7- to 9-year-old children at the commencement of instrumental training [3]. This author found that performance outcome depended on the amount of practice and general motivational level. It was also shown that intelligence and musical aptitude had no influence on outcome.

It is thought that this extraordinary intensity of musical training might shape the brain and its functions. Peculiarities in the brains of musicians have been reported on the grounds of morphometric analysis of post mortem brains, lesion data, and neuropsychological tests. However, only a few have searched for and found a relationship between the age of commencement of musical training and music-related motor skills or neuroanatomical peculiarities [4-6]. These studies strongly support the notion that extensive practice of musical functions could indeed shape the function and structure of the musical brain. A further research line has proposed that musicans may have an atypical pattern of hemispheric asymmetry with a deviation from normal leftward asymmetry of language functions towards rightward asymmetry or to a symmetrical organisation. However, the search for the cerebral hemisphere to which music is lateralised may be misguided. It rather appears that both sides of the brain are involved in musical functions and that interhemispheric and intrahemispheric information flow is of great importance for the maintenance of musical functions. Several approaches to studying brain functions have uncovered some pecularities in musical brains including larger anterior midsagittal corpus callosum, larger bilateral indices of hand motor areas, an expanded somatosensory hand representation in the left somatosensory cortex, reduced interhemispheric inhibition, or a superior spatial attention system in musicians to name but a few. Although these findings have furthered our understanding of the musical brain it is unclear how the different cortical and subcortical processing modules interact and how these interactions might be shaped by musical training. For example, we do know that the size of the hand motor areas (and the grey matter within these areas) of musicians are considerably enlarged, although there is no difference between musicians and nonmusicians in terms of overall brain volume. Thus, one might ask the question of whether the bilateral enlargement of the hand motor area has been accomplished at the expense of adjacent neural assemblies. That is, neurons normally responsible for the control of facial functions may also involved in hand motor control. It might also be the case that an expansion of the hand motor areas is accompanied by changing the local balance of excitation and inhibition within or between the areas, or it may well be a combination of all of these mechanisms.

The aforementioned example exemplifies the fact that it is not only interesting to study the particularity of a specific brain structure or function, but also the functional interaction between several brain regions or functions. The study of Lin et al. in this issue of Neuroreport [7] is a very good example of such kind of approach. The authors examined the influence of speech production (operationalized as reading aloud) on the modulation of the motor evoked potentials (MEP) induced in a specific finger muscle by transcranial magnetic stimulation (TMS) of the primary motor cortex in right handers. Interesting in itself, they found that the MEP in the right finger muscle was facilitated by reading aloud. Most interestingly, however, was the finding that in musicians (but not in non-musicians) there was also a facilitating effect in the left finger muscle of reading aloud. Thus, speech production had a facilitating influence on the non-dominant left hand motor system in musicians. Although this finding is important for the neuroscience of music because it reopens the question of whether musicans have atypical brain asymmetry for language functions, an interpretation on the basis of this experiment is not easy for several reasons. First, speech production is a relatively complex process involving several subprocesses comprising at least grammatical encoding, phonological encoding, a phonetic plan, the generation of a rhythmic structure, the articulation, and finally the feedback control of the generated speech output. Which of these subprocesses is responsible for the facilitating effect? 
Therefore, in future experiments it would be interesting to disentangle the particular subprocesses which might cause this facilitation effect. Second, it might also be the case that the facilitating effect is not an effect caused by speech production per se but rather by the fact that the subjects are doing something while they received TMS of the hand motor area. Thus, it would be necessary in further experiments to use tasks other than reading aloud in order to clarify whether it is speech production or one of the subcomponents which really is the critical process facilitating the MEP on the non-dominant side.

Although there are still some unanswered questions, the study of Lin et al. is a landmark study because it is the first to look at the functional interaction between different processing modules in musicians with TMS. This kind of research will definitely be an interesting and fruitful approach to delineating the functional modules of the brains of musicians and the brains of other excepional subject groups.

\section{REFERENCES}

1. Ericsson K, Krampe R and Tesch-Romer C. Psych Rev 100, 363-3406 (1993).

2. Sloboda J and Davidson J. The young performing musician. In: Deliege I and Sloboda J (eds). Musical Beginnings. Oxford: Oxford University Press; 1996.

3. O'Neill S. The role of practice in early musical performance achievement. In: Jorgensen $\mathrm{H}$ and Lehmann A (eds). Does practice make perfect? Current theory and research. Oslo: Norges Musikhogskole; 1997, pp. 53-70.

4. Amunts K, Schlaug G, Jäncke L et al. Hum Brain Mapp 5, 206-215 (1997).

5. Jäncke L, Schlaug G and Steinmetz H. Brain Cogn 34, 424-432 (1997).

6. Pantev C et al. Nature 392, 811-814 (1998).

7. Lin K-L, Kobayashi M and Pascual-Leone A. Neuroreport 13, 899-902 (2002). 